\title{
Upper Layer UAV Communication using LLR Algorithm for Flying Ad-hoc Network
}

\author{
Vidyashree D \\ Department of Electronics and Communication \\ Engineering \\ JSS Science and Technology University \\ (Formerly SJCE) Mysuru, India
}

\author{
Kavyashree M K \\ Department of Electronics and Communication \\ Engineering \\ JSS Science and Technology University \\ (Formerly SJCE) Mysuru, India
}

\begin{abstract}
Unmanned Aerial Vehicle (UAVs) has enormous potential in the network of regular citizen draws near, where humanoid ideally be at risk for eradication. An enormous swam of UAVs is used to give an adaptable and viable remote access coordinate with a huge inclusion region and better transmission rate for Internet of Things (IoT) services by means of hand-off hubs (relay nodes). The flying Ad-hoc Networks contain a group of little swam UAVs related in a specially appointed way, these incorporate line up into a crew to achieve ground-breaking objectives. In occasion of dreadful circumstances, if a standard communication system isn't accessible, these flying network systems are used to bear the rate of a self-serving, flexible, selfdesigning and tolerably inconsequential operational cost or simultaneously including various UAVs in a specially ad-hoc network system and force utilization and energy optimization are large difficulties. In this paper, energy optimization and the end to end delay, all other QoS parameters are proposed by utilizing an ideal total of upper-level layer UAVs as the hubs move separated and investigates it. Moreover, a Low Latency Routing Algorithm (LLRA) is used here to advance liveliness elementary for the UAV hubs and MAC layer adjust in transmission go and improves the quicker information processing rate and the end to end delay, throughput, packet delivery ratio, overhead. At long last, simulation outcomes shows the energy upgraded and utilized in UAV nodes and average connection delay which improves the packet transmission quantity than the conventional direction-finding algorithms.
\end{abstract}

Keywords- End to End Delay, Energy Optimization, Flying Ad-hoc Networks (FANETS), Low Latency Routing Algorithm (LLRA), MAC layer, Quality of Services (QOS), Unmanned Aerial Vehicle (UAV)

\section{INTRODUCTION}

The Unmanned Aerial Vehicle (UAVs) is a rising innovation that can be employed for fortified surveillance, timberland fire detection, and other non-military personnel applications so as to catch and convey an exact arrangement of information or data. In an improper calamity alert, where the regular network transmission arrange framework would be immobilized. These UAVs appears to serve by expanding the momentum of activity forms for sparing and recovery reason. In conditions like a harmful smoke infiltration, wildfires, and wild substantial following the UAVs are regularly sorted out by programming through a remote controller, towards the gracefully of restorative guide to zones which are disconnected and are less confused to cover an enormous territory without exhibitions in the security and insurance of the people groups catch.

As there is an upward in the prerequisites for a clear Quality of Service (QoS), the conventional cell organize takes an adequate measure of the period and they need flexibility in the presentation recompense and probability upgrades. IoT is one all the more advancing innovation and some imaginative creating Information Communication Technology (ICT, for example, the 5G gadgets/sensors spread an assortment of use fields. These sensor gadgets catch the information observing at the lower level layers that is ground level. In this way, these UAVs take the capacity to help the compact sensor to achieve a high-minded introduction and strip information stream of traffic start to finish postpone and streamline the force devoured by UAV hubs in the upper layer that is sky level.

This paper proposes a UAV connect with increasingly upgraded proficient force streamlining and considering starting to finish delay Quality of Service (QoS) parameter in the connection of the system improves life expectancy, energy usage, and drooping in packet reachability. Shows how it is conceivable to improve the unfaltering quality of course.

Technological gaps or Challenges of UAV as there is consistently a tradeoff between Payload limit and flight time in drone innovation. Routinely, locally available lightweight lithium-particle batteries are utilized to flexibly capacity to $\mathrm{UAV}$, yet their capacity reinforcement isn't practically identical with different batteries. With the expansion in payload, continuance diminishes and consequently, the mission may not get its finishing. Fixed-wing drones are effective in power use, however, they have the disadvantage of floating and speed control. Flying a solitary automaton may likewise experience a flight disappointment because of some mechanical and climatic reasons, so there is consistently a need to give reinforcement. Up and coming multitude trip of automatons can execute this assignment, where if there should be an occurrence of disappointment of one automaton, others complete the mission. This innovation is reliant on Swarm movement of creepy crawlies, ants and winged animals and utilizes man-made brainpower, yet in creating stage. Automatons are as yet constrained by controlling through human administrators, incorporation of Artificial Intelligence will permit an automaton to settle on 
brilliant choices and work in like manner rather than human controllers.

Automatons are as yet restricted by controlling through human administrators, incorporation of Artificial Intelligence will permit an automaton to settle on keen choices and work in like manner rather than human controllers. Potential gains and damages are yet to be investigated in this direction. Automatons likewise experience the ill effects of breezy conditions and unfriendly atmosphere changes. Splashing Drones are effective to shower less territory, however for mass splashing, they become less proficient, and the activity cost turns out to be high.

\section{LITERATURE SURVEY}

In the writing overview, there are numerous calculations utilized over the world to improve the exhibition Quality of Services Parameters and vitality streamlining. In one paper Aforementioned Algorithm has been utilized for differing learning contraptions so as to catch live varying media spilling information move which has a significant impact in the Industrial-Internet of Things (IIOT) applications and this likewise raises the number of courses that is fundamental to trade of information correspondence. They have utilized the 2-tired heterogeneous remote system which incorporates more sensors with restricted battery life. To gauge the boundary imperatives, they have used the MATLAB device. The proposed methodology improves the vitality utilization and regular deferral on the dis-secured courses while in transit to their endpoint. Moreover, gives a short report for $5 \mathrm{G} / \mathrm{IoT}$ incorporation advancements with sensors [1]. This overview paper presents an outline for the reserving of UAVempowered little cell arrange frameworks. The scattered storing strategy for UAVs has likewise introduced. This paper, for the most part, clarifies the 3 issues on how UAV offers information offices to versatile clients with backhaul relations. At that point, battery-worked limitations as UAV need not rely upon the fixed force flexibly. This paper consolidates the UAV and storing to advance the presentation boundary issues of little cell arrange lattices. A contextual investigation of Interference additionally offered [2]. This article depicts a bound together plan for UAV bolstered zones like a natural fiasco. It focusses on how direction correspondence planning on UAV is limited for the versatile client getting by on the ground level. A multi-bounce Device to Device and multi-jump transmitting plan is used to upgrade areas of UAVs. This paper explains the communication efficiency of UAV standard enhancement Algorithms and the Shortest Path Routing (SPR) Algorithm used to set up and extend the unbeatable course and to improve the transmit issues [3]. Low Latency Routing Algorithm (LLRA) is used to achieve the ideal course way with the least deferral (slack) and enthusiastically distribute information traffic flows. This Layered UAV gathering of swam arrange topology design anticipated and the best amount of UAVs are inspected. The daily schedule of this LLRA is demonstrated to propel the bundle move proportion [4]. The Fast Explore and Exploit Learning (FEEL) Algorithm is executed to VR submersion and this paper validates the arranged VR presentation viability and in moved
VR inundation fideli-ty, application intuitiveness/play-out potential, and communicate power ingesting [5]. TPA-FCP Algorithm and Polynomial-time Randomized Approximation Scheme are used to determine the general time in ideal direction estimation yet the UAVs are associated base station over a straight system association and the anticipated framework here gives 4 joined segments relying on the degree to cutting edge shows the advancement in the UAV vitality utilization capability to propose blaming course streamlining issue in a huge scope network framework [6]. Multi-Layered Planning is used to allow vitality compelling uplink correspondence for engaging open insurance and security interchanges, which has the ability to allow correspondence at ground level by methods for wired or remote correspondence. Poly-nomial-time Randomized Approximation Scheme (PRAS) introduced to find the minimal number of drifting areas [7]. Time Division Multiple Access (TDMA) is used to allow comparison to communicates and exhibitions in the UAV-helped system. This plan gives an expectation to minimalize the general force utilization of UAV [8]. The iterative calculation is used to determine the subproblems and the general correspondence power for gadgets is minimalized beneath the Signal-ToInterference-Plus-Noise-Ratio. The general correspondence intensity of the IoT gadgets is dense by $45 \%$ [9]. To propel the QOS boundaries by considering the 3-tire cross breed organize as UAV helps out satellite telecom and base station. Low-multifaceted nature insatiable inquiry calculation and Lagrange double breakdown and inwardly curved procedure (CCP) method are used and execution of saving arrangement collects an upgrade in the UAV system's overhead and throughput [10]. Fly-float and-correspondence Technique is acquainted with deciding the exchange off issue in the existing plan and divergent rules for situations perfect elevation and beamwidth gauges in assorted multiuser imitations and it bears helpful recognition for UAV correspondence arrange [11]. Non-Orthogonal Multiple Access (NOMA) and combi-country outline portrayal. Here it likewise gives the practicality examination and procedure for vitality efficient height enhancement and vitality power conveyance, and this plan achieves up to $18 \%$ force saving money on signal communicate with extraordinary influence redeemable [12]. The two-phase calculation and three-phase elective algorithms are utilized in paper [13] to break the issue of gravely carried on fractional and binary calculation offloading strategies and it additionally gives the structure for asset distribution plots beats and the diagrams unite is wild and has a less computational confusion. This [14] paper fundamentally clarifies the computerized scientific approach utilized for dissecting the information caught by automatons and they proposed a self-sorting out guide to give the guide to destitute one. This [15] proposed another casing chip away at versatility the executives alongside the transmission force and speed of the spine hub. In this portability, the model has been made with a one-jump separation between a wide ranges of hubs. The system is worked with 2 modes on is the spine with moderate speed and ordinary hub without any restrictions for speed. This paper, for the most part, handles with speed levels with high transmission spines. 


\section{PROBLEM STATEMENT}

As there are various bearing discovering conventions proposed for Mobile Ad-hoc net-works every one of them has its highlights, benefits, and negative marks for singular application conditions. At the point when the circumstances are too intricate to even consider accomplishing and if the network geographies arrangement changes rapidly, the individual course finding proto-col won't experience the important requests of differed customizable hubs nor can ensure the system routinely. Exact knowledge of the neighboring area and reasonable change approaches of UAV hubs have built up the propensity to receive diverse bearing discovering conventions with aggregate supplies of Quality of Services (QoS) parameters.

\section{A. Existing System}

By considering all the referenced papers in the writing study. The current framework is finished up as in the current century, the capacities and jobs of Unmanned Aerial Vehicles (UAVs) have immediately changed, and the training in equipped and non-military personnel zones is colossally mainstream because of the advancement in the innovation of machine-like automated mechanical frameworks like workstations, sensors gadgets, transport organize, and organizing bits of information. Albeit Mobile Ad-hoc Networks takes a multireason application there's a necessity of specific information that might be overpowered from genuine where obsolete MANET doesn't appear to be usable like disaster circumstances like sinking or equipped fight zone. It's impractical to place in transportable hubs (which travel outward) in such a correspondence zone.

\section{B. Proposed System}

A secured UAV layered system upper-layer engineering is utilized and researched in this proposed framework and the ideal aggregate of UAVs in the unrivaled layer is inspected with contiguous structure presentation fringes. In addition, all outnumber of $20 \mathrm{UAV}$ hubs is put at the tallness of 50-90 meters to organize engineering development. The low idleness directing calculation (LLRA) is used by utilizing data about the area balances of hubs and system availability of UAVs in FANETs. Because of the minutest slack in delay and the anticipated association dauntlessness of UAVs, the utilized LLRA can achieve the ideal way with the less defer and vivaciously assign countless information traffic streams, which can capably misuse the parcel move proportion and advance the unfaltering quality of direct way and improves the normal measure of vitality upgraded.

\section{METHODOLOGY}

These days, a large portion of the Internet of Things (IoT) gadgets utilize the current innovation the Unmanned Aerial Vehicles (UAVs) that are introduced or sent proficiently to convey high Caliber of Services. This paper focuses on the Flying Ad-hoc Networks (FANETS) correspondence innovation for numerous swam of UAVs to cover the gigantic inclusion territory of IoT gadgets administrations. In this way, the UAVs can ensure correspondence execution during a catastrophe condition without the cooperation of the individual. The UAV hubs set at various topographical areas are utilized to catch the data or information starting from the earliest stage from any IoT gadgets/sensors as that is conceivable utilizing an ordinary customary system. As the low inertness administration prerequisite while sharing the data in those crisis circumstances bring UAV to arrange geography as it changes without fail and vitality devoured by every hub gets expanded as geography changes once in a while.

To address the end to end lag in time and energy or aliveness of nodes improvement accomplishment. The underneath Figure 1 shows the high-level overview of how the functionality and responsibility of the layered UAV hubs utilize the Low Latency Routing Algorithm (LLRA) to screen the calamity circumstance by sharing among each UAV hubs lastly sending it to the Base Station (BS) utilizing remote correspondence. The IoT sensor gadgets or UAVs catch the data from the lower ground level during calamity circumstances. Those catch data from ground level is then moved to the closest steering UAV hub for information correspondence lastly handled to the goal UAV hub and afterward will be moved to the Base Station (BS). Where the area in the calamity condition needs help (clinical if necessary).

The Low Latency Routing Algorithm (LLRA) is used to utilize the data about the area dependent on the balances of hubs and system network of UAVs. This calculation fundamentally decides the strategy to find the course to send information parcels from the underlying to definite goal UAV hubs in the upper layer network of FANETs. In light of the adjusting of the MAC layer is utilized in a transmission range to improve the quickest information handling rate and limit the deferral in UAVs hubs interface solidness. The anticipated framework utilizes LLRA, which is utilized to achieve the ideal course with the base postponement and progressively balance the information streams, which can productively limit the normal measure of vitality used by UAV hubs and improve the dependability of a course. This calculation is utilized here to control the congestion of parcels in the system and lifts the presentation of start to finish delay, as not all the UAV hubs of the system add to the course revelation for a particular beginning source hub to conclusive goal hub pair up. Defer obtuse gauges, for example, $802.11 \mathrm{~b}$ and $802.11 \mathrm{~g}$ can work with various information rates for QoS-compelled correspondence to use the halfway assets of UAV increasingly able.

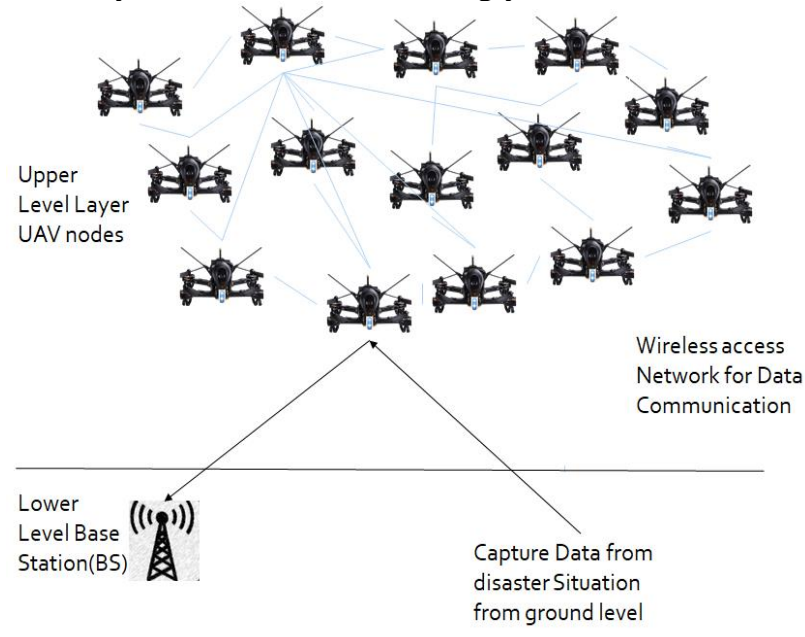

Figure 1: Layered UAV hubs cooperating with BS for data traversal 


\section{SYSTEM DESIGN}

The System design describes the general procedure and components which are involved. It demonstrates how the complete structure is separated as a subsystem, all deals through one or many data or controller runs to or from all other, also together provides all functionality of scheme as entire. This is used to identify the inner data, it must exist in association to do its job, also demonstrates the flow of the data among various portions of associations.

Software development is generally a stepwise process. Before the way toward executing the developed system, it includes the procedure of programming plan. A Software configuration is a depiction of the structure of the product to be actualized, the information which is a piece of the framework, the interfaces between the segments, once in a while, the calculations utilized. Architects don't show up at a completed structure quickly yet build up the plan iteratively through various renditions. The plan procedure includes including custom and subtleties as the structure is created, with consistent backtracking to address prior structures.

In numerous software advancement schemes, programming configuration is an ad-hoc procedure. Gazing from the arrangement of prerequisites, for the most part in common language, a casual structure is readied. Coding initiates and the structure stage is changed as the framework is executed. At the point when the execution stage is finished, the structure has typically changed such a great amount from the underlying particular that the first plan record turns into an off base and inadequate depiction of the framework.

There are several advantages of the design phase. Some of them are listed below:

- The plan stage assists with understanding the client necessities and assists with planning the client prerequisites into the execution stage.

- The cycles in the plan stage help to fuse however many client prerequisites as would be prudent in the last programming being created.

- The structure stage lessens the cost associated with the advancement of the product the same number of changes would be made to the product in the execution if the plan isn't clear.

The structure procedure is iterative and requires thought of different plan choices at each stage. The target of the structure stage is to create the general plan of the product.

The Below Figure 2 is the system design that explains the flow of the general procedure that are involved in the development of proposed system. Firstly, the initialization network along with the construction of topology for the network of UAV nodes. Then route calculation through LLRA algorithm using link disjoint and MAC parameters which the job of faster data processing for efficient delivery of informtion form source to destination routing through relay nodes. Then final step is performance parameter analysis i.e all QoS parameter are checked.

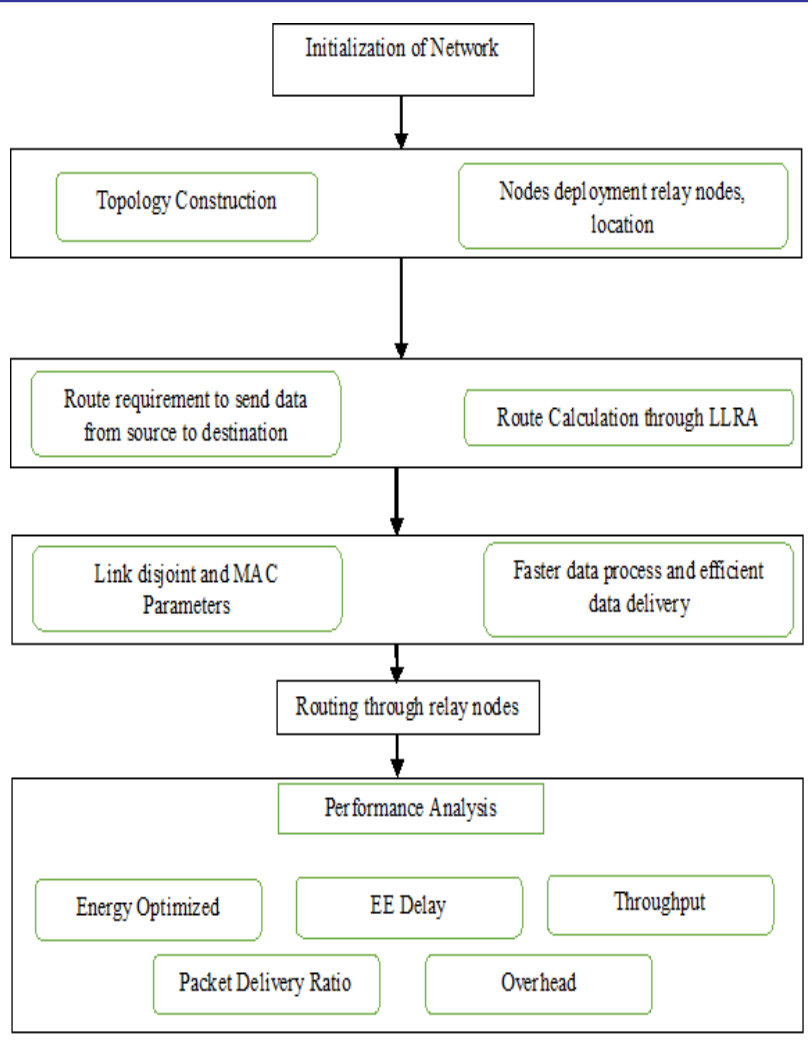

Figure 2: System design of complete model of proposed system

\section{IMPLEMENTATION}

The implementation stage of any schema improvement is the utmost significant segment as it yields to the conclusion of the system, which resolves the problem at needle. This execution stage includes the real appearance of the thoughts, which are communicated in the investigation report and created in the structure stage. Usage ought to be ideal planning of the structure record in an appropriate programming language so as to accomplish the vital last item. Regularly the item is demolished because of off base programming language picked for execution or inadmissible strategy for programming. It is better for the coding stage to be legitimately connected to the structure stage in the sense on the off chance that the plan is regarding object arranged terms, at that point usage ought to be ideally completed in an item situated manner.

There are three major execution verdicts that have been made before the implementation of this project. They are as follows:

i. Selection of the platform (Operating System).

ii. Selection of the programming language for development of the application.

iii. Coding guideline to be followed.

The implementation stage in this proposed method involves certain implementation modules that are:

1. Network Module

2. Energy Module

3. LLRA

4. Signal to Noise Ratio SNR) and Link Quality Indicator

5. Neighbor Discovery

6. MAC layer Parameters 


\section{Network Module}

This part contains a picture of the functionality of the contents utilized in building topography. This module includes building Wireless Network layout, topography comprising of versatile mobile hubs, every node employed with different channels.

This module comprises of following advances:

i. Setting up Wireless Network Topology: This incorporates ecological settings, hub/node setup, and layout/topology creation.

ii. Setting the transfer speed and edge: Each and every hub in the system geography will be doled out with certain data transmission and geography.

iii. Identifying the neighbors: In request to distinguish the neighbors for a specific hub, the Euclidian separation idea is utilized.

iv. Specifying the information transmission through single and multi-jump: From which hub the information must be sent and which hub must get the information will be determined. Additionally how many measure of information must be sent alongside the time frame the information will be indicated.

v. Specifying the recreation start time and end time: In NS 2 the whole exchange happens inside a portion of seconds. The exchange can be seen through the NAM window whenever. For this, the reenactment start time and end time will be indicated.

Algorithm 1. Node Deployment Algorithm

1: Procedure initialization of nodes randomly

2: for early node to ultimate number of nodes

3: initialize the portable nodes

4: Nodes Energy

5: Set the initial position for portable mobile nodes/hubs

6: Initialize the agent

7: Attach agent to node

\section{8: End for}

\section{Energy Module}

Energy Model, as executed in, is a hub/node quality. The energy model speaks to level of vitality in a flexible host. The energy model in a hub has an underlying worth which is the degree of liveliness the hub has toward the start of the modernization. This is known as initial Energy_. It additionally has a given energy utilization for each parcel it transmits and gets. These are called transmit power (txPower_) and receive power (rxPower_).

The energy model just keeps up the absolute liveliness and doesn't keep up radio states. It is conventional enough for future reenactments, for example, the CPU power utilization. Kindly note that the old vitality model without a doubt keeps up some radio states, and have a few techniques to control them, and they are just utilized by the versatile devotion module. This methodology may cause irregularity with remote phy. To keep versatile loyalty work, we didn't expel it from the vitality model, yet it is out of date, and ought not to be utilized further. Presently all entrances to the vitality model should experience remote phy.

The energy exemplary is used over the node-config API. An example is shown as given in below Figure 3:

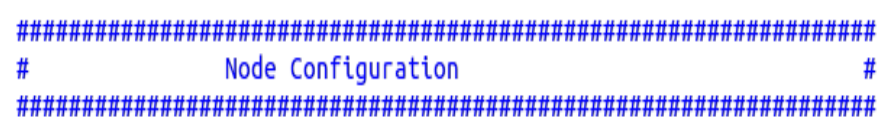

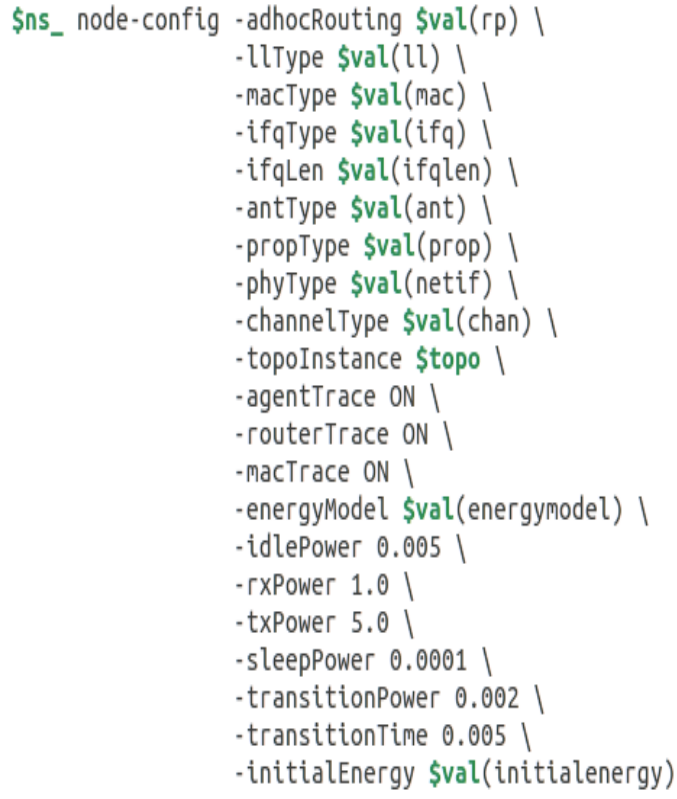

Figure 3: Node Configuration API for Energy

Algorithm 2. Node Energy Calculation Algorithm

1: Procedure initialize nodes with initial energy in Joules and random node deployment with energy

2: for early node to ultimate number of nodes

3: Compute the energy consumed by each node as $\mathrm{E}=\mathrm{E}_{\mathrm{tx}}-\mathrm{E}_{\mathrm{tr}}$

4: Calculate node residual Energy = initial energy-

Consumed energy

5: Calculate the same for all nodes

6: All nodes will be created at same location in topology and creates random node position

7: Set size RNG

8: set randX, rand $\mathrm{Y}$ as number of nodes

9: End for

\section{LLRA}

Considering the coverage limits of each UAV in the upper layer constrained by the transmission power, several upper layer UAVs should cooperate with each other to guarantee an effective full coverage to lower layer UAVs. In order to minimize the communication latency and multiple relay hops in the proposed layered UAV network architecture, how to design the appropriate number of upper layer UAVs as relay nodes is a challenging coverage optimization problem. Therefore, the layered UAV network design problem is defined as how to deploy the minimum number of UAVs in 
the upper layer to realize an effective full coverage to lower layer UAVs.

\section{Signal to Noise Ratio SNR) and Link Quality Indicator}

It is characterized as the distinction in decibel between unadulterated got signal quality and the clamor floor. SNR can give a superior gauge of connection quality contrasted with RSSI. Be that as it may, SNR just separates between excellent connections and different connections i.e., it doesn't give a more extensive territory to interface quality characterization. What's more, because of radio miscalibration, SNR can prompt uproarious connection quality gauge.

The LQI estimation is a portrayal of the quality and nature of the got bundle. The estimation might be executed utilizing SNR or recipient vitality level or a mix of these strategies

\section{MAC Layer Parameters}

One-to-one channel mapping mechanism between channel state field and multiple channels is proposed to convey available channel number. Nodes can obtain available frequency by regular beacon frame reception. Instead of keeping a channel list, mapping rules help to save energy for energy-limited nodes. An efficient channel selection strategy to organize the nodes to access separate channels.

An efficient channel selection strategy to organize the nodes to access separate channels. Taking full account of nodes' priority, a coping strategy is designed to deal with the channel selection conflict

Below Figure 4 is the flow chart that explains the flow of the working model from the initialization step to the output result of data routing to the destination using the LLRA algorithm. Firstly, all the initialization simulation parameters will be initialized along with the no of UAV nodes used. Then the routing starts from the source node initialized. While routing using the LLRA routing algorithm with the MAC parameters checks for the shortest path to the destination node by the selected relay nodes. Checks for the minimum delay if yes then calculates the average energy by the nodes and the End-to-End Delay by calculating the route with minimum selected relay nodes through this data processes faster with efficient delivery of data to the destination node. The average packet delivery ratio is calculated by using the ratio of number of received and sent along with the lost packets. The average throughput of overall proposed system from initial to end time.

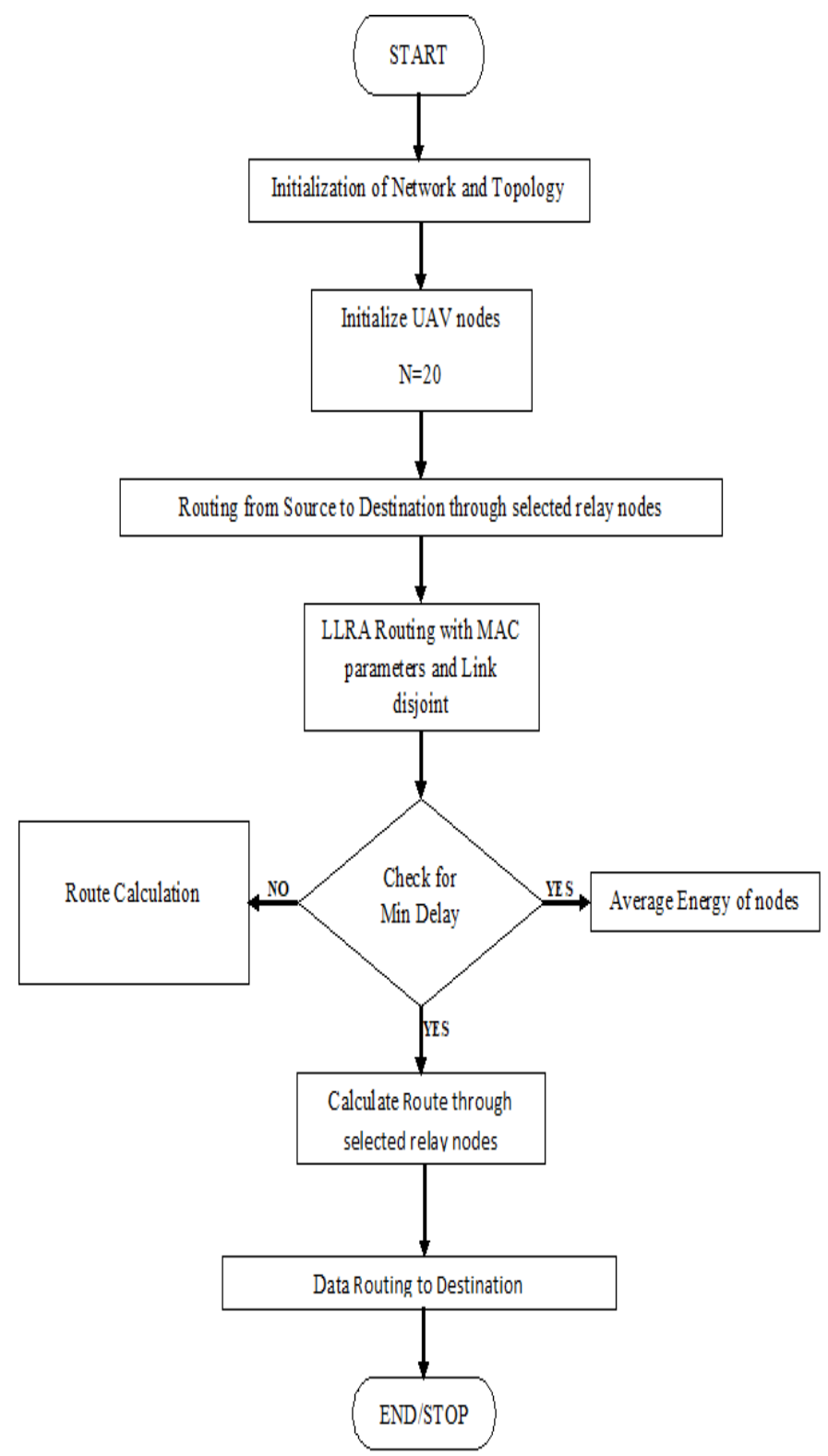

Figure 4: Flow Chart of Proposed System

The Implementation of the proposed system in the NS2 Platform has simulation parameter settings that need to be done before setting up the system. The Figure 5 and the Table 1 Shows the simulation parameters values. Where the channel is set to the wireless channel, the TwoRayGround is a radio propagation model that depicts the path loss between the source and the destination node. Then the network inter-face is also wireless. The MAC layer used is 802.11 with the Drop-Tail interface queue. The overall area considered for the implementation of the proposed system is $800 \times 800$. The total number of UAV nodes is 20 and the impact height of UAV will vary from $50-90$ meters. $512 \mathrm{~K}$ bytes of packets will be forwarded with the queue window is 32 bytes using TCP protocol using a wireless network interface at a constant bit rate. 


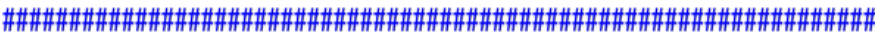 \\ $\# \quad$ Setting the Default Parameters}

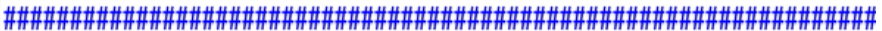

\begin{tabular}{|c|c|}
\hline set val(chan) & Channel/Wirelesschannel \\
\hline set val(prop) & Propagation/TwoRayGround \\
\hline et val(netif) & Phy/wirelessPhy \\
\hline et $\operatorname{val}(\mathrm{mac})$ & $\mathrm{Mac} / 802 \_11$ \\
\hline t val(ifq) & Queve/DropTail/Priqueve \\
\hline t val(ll) & LL \\
\hline t val(ant) & Antenna/OmniAntenna \\
\hline set $\operatorname{val}(x)$ & 800 \\
\hline set val(y) & 800 \\
\hline set val(ifqlen) & 80 \\
\hline et val(nn) & 20 \\
\hline set val(stop) & 100.0 \\
\hline et val(rp) & LLRA \\
\hline set $\operatorname{val}(c p)$ & "20" \\
\hline set $\operatorname{val}(\mathrm{sc})$ & "tcp" \\
\hline t val(energymodel) & EnergyModel \\
\hline t val(initialenergy) & ;\# Initial energy in Joules \\
\hline
\end{tabular}

Figure 5: Experimental settings of NS2 Platform

TABLE 1. DEFAULT SIMULATION PARAMETERS

\begin{tabular}{|c|c|}
\hline Simulation Parameters & Values \\
\hline NS Version & NS2 \\
\hline Network Area & $800 \times 800$ \\
\hline Channel & Wireless Channel \\
\hline Interface Queue & Drop Tail \\
\hline No. of Nodes & 20 \\
\hline MAC Layer & 802.11 \\
\hline Traffic & CBR \\
\hline Transport Protocol & TCP \\
\hline Network Interface & Wireless \\
\hline Packet Size & $512 \mathrm{~Kb}$ \\
\hline Queue Window & 32 \\
\hline
\end{tabular}

\section{RESULTS}

The below snapshots Figure shows the upper layer (sky level) UAV hubs/nodes transmitting information from source to end node where source and destination are node 3 and 17 respectively. Figure and Figure shows the average amount of energy-optimized and end-to-end delay, throughput, packet delivery ratio and overhead calculated using the Network Simulator (NS-2). Figure - Figure are the X-graph which shows the graphical representation of the values achieved by the recommended scheme along with the existing system.

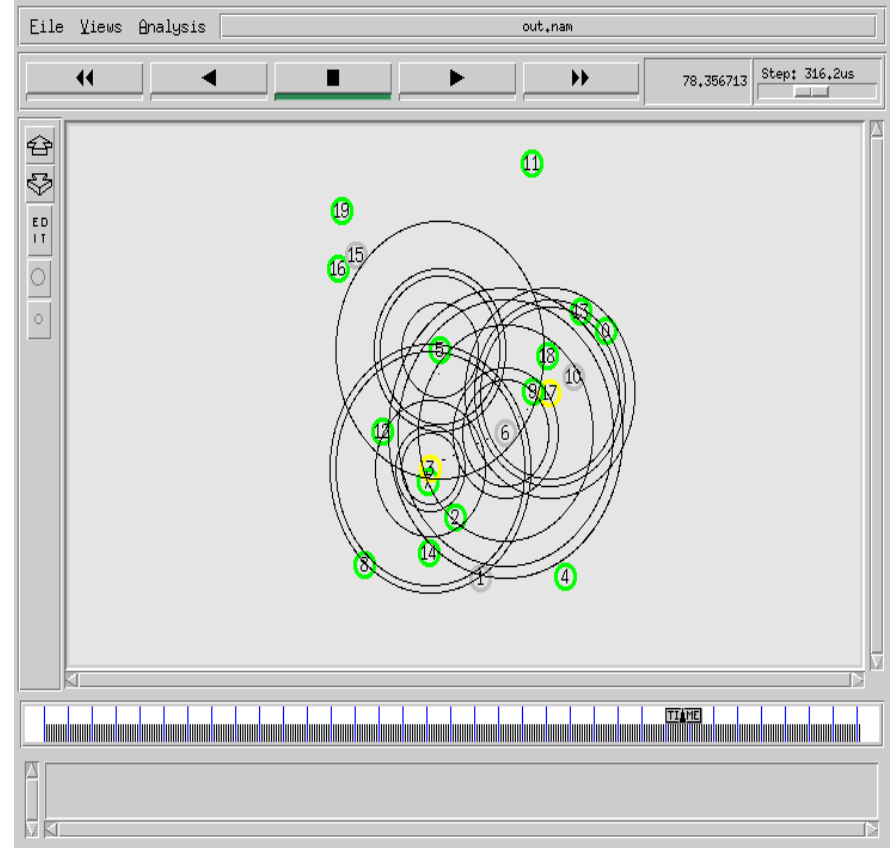

Figure 6: It shows the source UAV upper layer nodes transmitting data to destination UAV node.

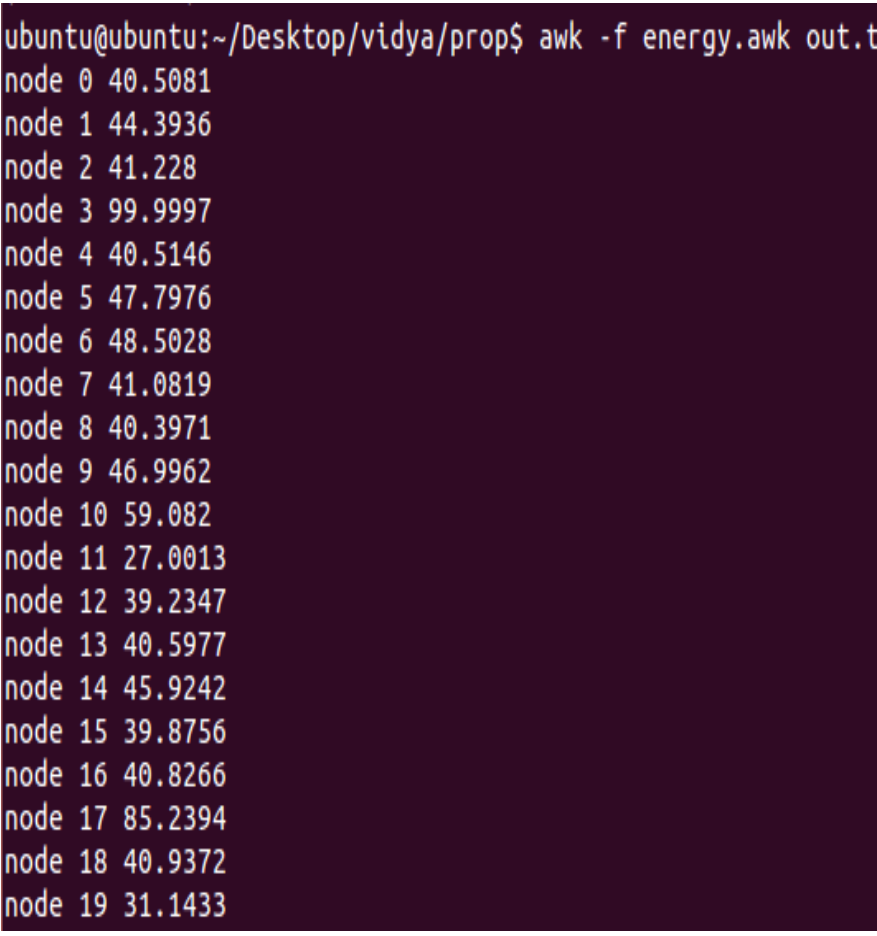

Figure 7: Shows the amount of energy consumed by each UAV nodes

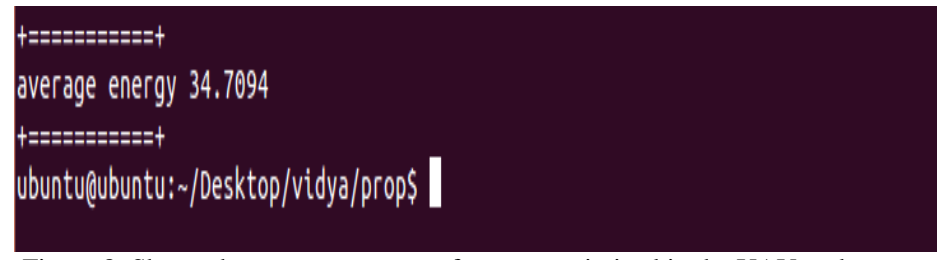

Figure 8: Shows the average amount of energy optimized in the UAV nodes while transfer of data 
ubuntuaubuntu; /Desktop/vidya/prop\$ awk - f ezedelay, awk out, tr

\section{Average End-to-End Delay $=72.4058$ ms}

ubuntuQubuntui /Desktop/vidya/prop\$ awk -f throughput. awk out.tr

Average Throughput[kbps] $=415.94$

StartTime $=10.00$

97.55

ubuntuQubuntu; / Desktop/vidya/prop\$ awk - f overhead, awk out.tr

Overhead $=3.156$

UbuntuQubuntu: /Desktop/vidya/prop\$ awk - f pdf. awk out.tr

s:17905 r:17706, r/s Ratio:0.9889, f:8119 loss:199

ubuntugubuntu: / Desktop/vidya/prop\$

Figure 9: It shows the average amount of end-to-end delay, throughput and PDR (Packet Delivery Ratio) and overhead accomplished at the multiple relay UAV nodes and traversal of data from one node to other

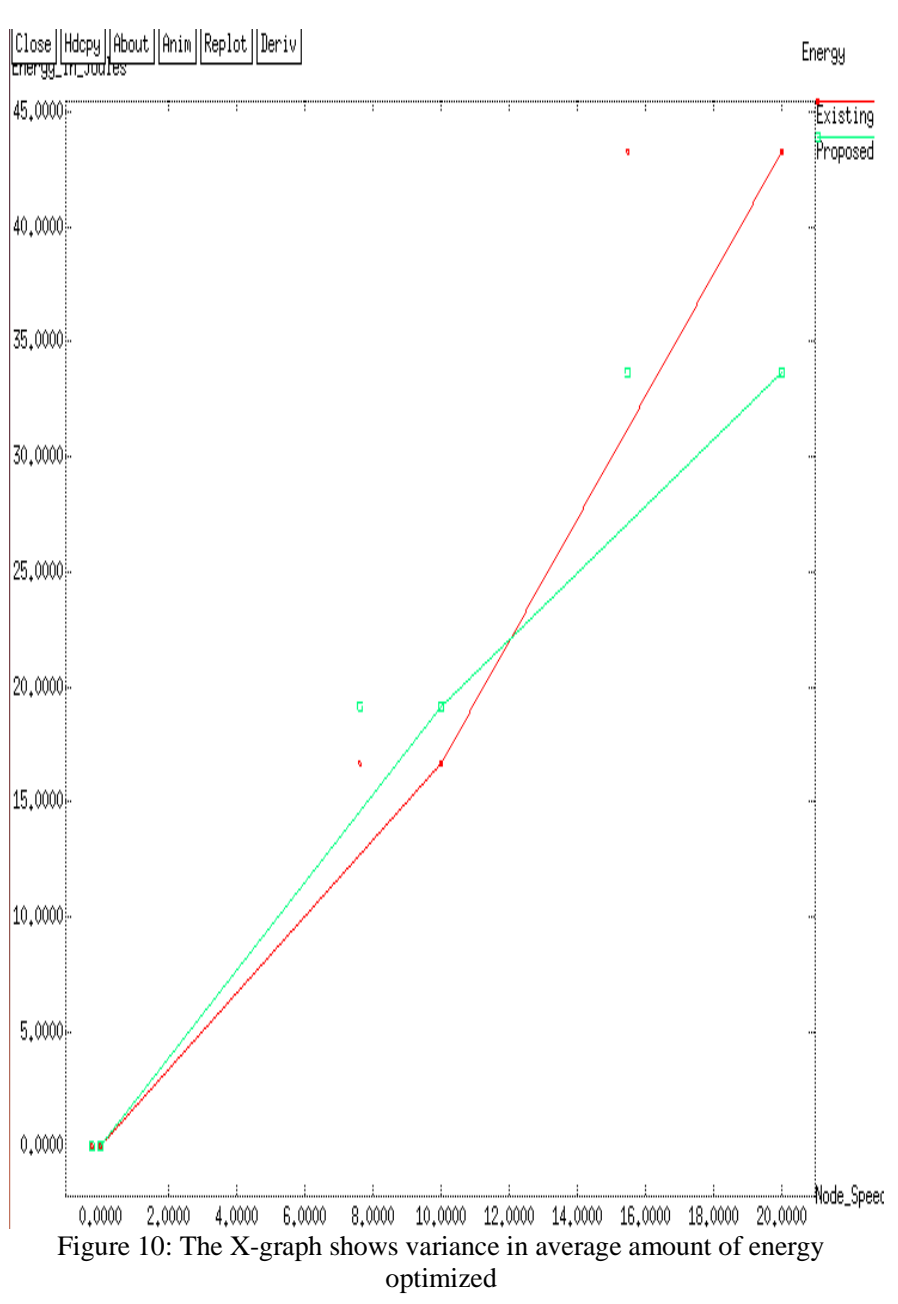

Figure 10: The X-graph shows variance in average amount of energy optimized

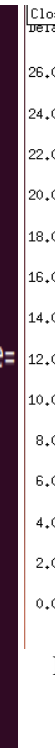
Delay

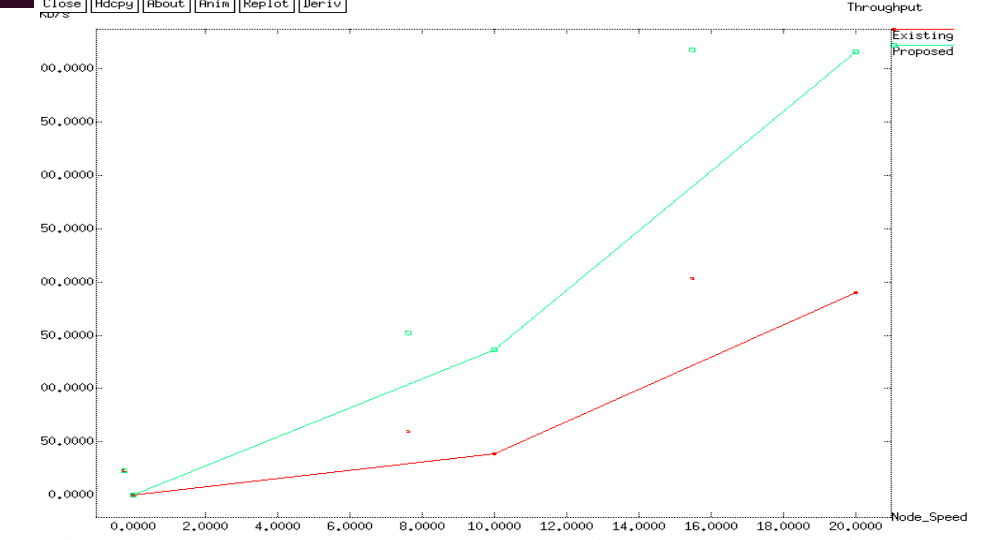

Figure 12: The $\mathrm{X}$-graph shows average amount of throughput achieved from existing to proposed system

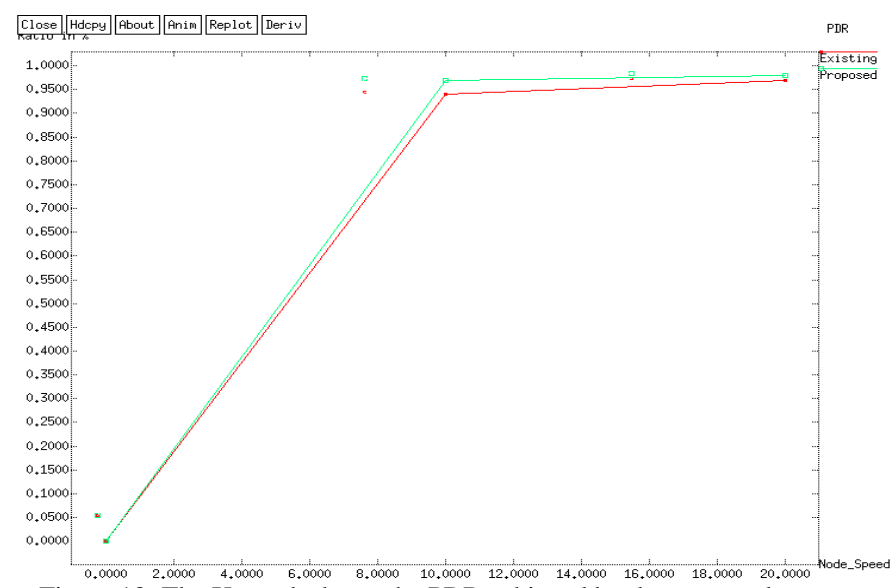

Figure 13: The X-graph shows the PDR achieved by the proposed system 


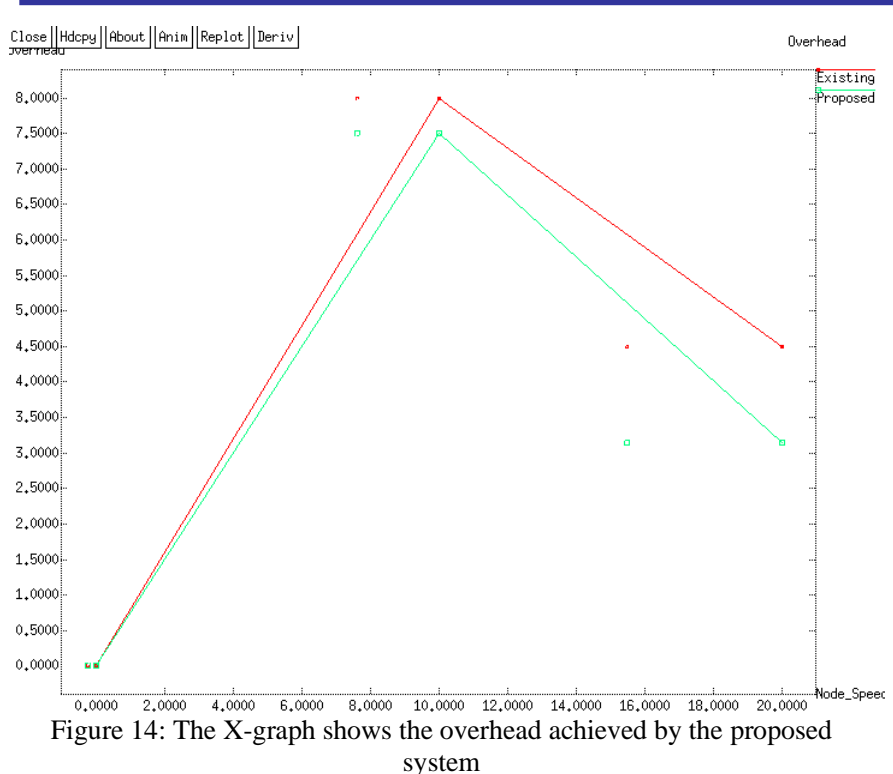

TABLE 2. PARAMETER VALUES TABULATED

\begin{tabular}{|c|c|}
\hline Parameters & Values \\
\hline Average Energy & $34.7094 \mathrm{~J}$ \\
\hline Average End-to-End Delay & $72.4058 \mathrm{~ms}$ \\
\hline Average Throughput & $415.94 \mathrm{Kbps}$ \\
\hline PDR ratio & 0.9889 \\
\hline Overhead & 3.156 \\
\hline
\end{tabular}

The above Table 2 gives the values that are predicted by using the projected layered network architecture by using LLRA algorithms this table also shows the average amount of energy-optimized, end-to-end delay, overall throughput, and the packet delivery ratio (PDR), and overhead achieved.

\section{CONCLUSION}

This paper proposed to keep away from a unique method to bring an unfailing scheme of execution with a FANET brought together a technique for network accomplishment. To accomplish the most ideal courses in position condition the energetic UAV hubs in the system design are used. In this anticipated strategy, the MAC layer constraints are utilized check-up in transmission extend for quicker information processing rate which likewise ensures dependability on directing routes, and load appropriation is through to decrease the traffic over-burden among the neighboring UAV hubs and communication links by investigating end to end delay. At long last, the average energy utilization is about $\mathbf{J}$ and end to end delay is decreased to $70.40 \mathrm{~ms}$ for $20 \mathrm{UAV}$ upper layer nodes. If there should arise an occurrence of hub disappointment self-recouping and self-adjustment are utilized to rule the courses more than once and keep on communicating. Energy heedful navigation calculation alongside LLRA to improve the average amount of energy, packet delivery ratio, overhead updated.

\section{ACKNOWLEDGMENT}

I tremendously thankful for Dr. Shankaraiah, Head of the Department of Electronics and Communication Engineering, JSS Science and Technology University (Formerly SJCE), Mysuru, for providing me timely suggestions, encouragement and support throughout the project. I'm indebted for Asst. Prof. Kavyashree M K, Department of Electronics and communication, JSS Science and Technology University (Formerly SJCE), Mysuru, for providing her valuable guidance, constant assistance and constructive suggestions for effectiveness of project.

\section{REFERENCES}

[1] Fadi Al-Turjman, Sinem Alturjman, "5G/IoT-enabled UAVs for multimedia delivery in industry-oriented applications," Springer Science, 2018.

[2] Nan Zhao, F. Richard Yu, Lisheng Fan, Yunfei Chen, Jie Tang, Arumugam Nallanathan, "Caching UAV-Enabled Small-Cell Networks", 2018

[3] Nan Zhao, Weidang Lu, Min Sheng, Yunfei Chen, Jie Tang, F. Richard $\mathrm{Yu}$, Kai-Kit Wong, "UAV-Assisted Emergency Networks in Disasters", IEEE Wireless Communications, volume 26, issue 1, 2019.

[4] Qixun Zhang, Menglei Jiang, Zhiyong Feng, Wei Li, Wei Zhang, Miao Pan, "IoT Enabled UAV: Network Architecture and Routing Algorithm" IEEE Internet of Things Journal, volume 6, issue 2, 2019.

[5] Jacob Chakareski, "UAV-IoT for Next Generation Virtual Reality", IEEE Transactions on Image Processing, volume 28, issue 12, 2019.

[6] Pengfei Wu, Fu Xiao, Chao Sha, Haiping Huang, Lijuan Sun "Trajectory Optimization for UAVs' Efficient Charging in Wireless Rechargeable Sensor Networks", IEEE Transactions on Vehicular Technology, volume 69, issue 4, 2020.

[7] Shanza Shakoor, Zeeshan Kaleem, Muhammad Iram Baig, Omer Chughtai, Trung Q. Duong, Long D. Nguyen, "Role of UAVs in Public Safety Communications: Energy Efficiency Perspective", IEEE Special Section on Mission Critical Sensors and Sensor Networks, volume 7, 2019.

[8] Yao Du, Kun Yang, Kezhi Wang, Guopeng Zhang, Yizhe Zhao, Dongwei Chen, "Joint Resources and Workflow Scheduling in UAVEnabled Wirelessly-Powered MEC for IoT Systems", volume 68, issue $10,2019$.

[9] Mohammad Mozaffari, Walid Saad, Mehdi Bennis, Merouane Debbah, "Mobile Unmanned Aerial Vehicles (UAVs) for Energy-Efficient Internet of Things Communications", IEEE Transactions on Wireless Communications, volume 16, issue 11, 2017.

[10] Jingjing Wang, Chunxiao Jiang, Zhongxiang Wei, Cunhua Pan, Haijun Zhang, Yong Ren, "Joint UAV Hovering Altitude and Power Control for Space-Air-Ground IoT Networks", IEEE Internet of Things Journal, volume 6, issue 2, 2019.

[11] Haiyun He, Shuowen Zhang, Yong Zeng, Rui Zhang, "Joint Altitude and Beamwidth Optimization for UAV-Enabled Multiuser Communications", IEEE communication, volume 22, issue 2, 2018.

[12] Muhammad Farhan Sohail, Chee Yen Leow, Seung Hwan Won, "Energy-Efficient Non-Orthogonal Multiple Access for UAV Communication System", IEEE Transactions On Vehicular Technology, volume 68, issue 11, 2019.

[13] Fuhui Zhou, Yongpeng Wu, Rose Qingyang Hu, Yi Qian, "Computation Rate Maximization in UAV-Enabled Wireless Powered Mobile-Edge Computing Systems", IEEE Journal on Selected Areas in Communications, volume 36, issue 9, 2018.

[14] Sri Harsha Mekala, Zubair Baig, "Digital Forensics for Drone DataIntelligent Clustering Using Self Organising Maps", International Conference on Future Network Systems and Security, pp. 172-189, Australia, 2019.

[15] Smys, S., G. Josemin Bala, and S. Jennifer. "Mobility management in wireless networks using power aware routing"; International Conference on Intelligent and Advanced Systems, pp. 1-5. IEEE, 2010. 